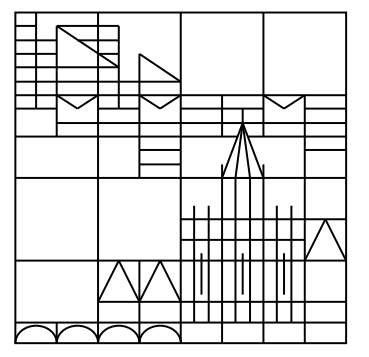

\title{
Maximal Regularity and Asymptotic Behavior of Solutions for the Cahn-Hilliard Equation with Dynamic Boundary Conditions
}

\author{
Jan Prüss \\ Reinhard Racke \\ Songmu Zheng
}

Konstanzer Schriften in Mathematik und Informatik

Nr. 189, Juni 2003

ISSN 1430-3558

Konstanzer Online-Publikations-System (KOPS)

URL: http://www.ub.uni-konstanz.de/kops/volltexte/2007/2216/

URN: http://nbn-resolving.de/urn:nbn:de:bsz:352-opus-22160

(C) Fachbereich Mathematik und Statistik

(C) Fachbereich Informatik und Informationswissenschaft

Universität Konstanz

Fach D 188, 78457 Konstanz, Germany

Email: preprints@informatik.uni-konstanz.de

WWW: http://www.informatik.uni-konstanz.de/Schriften/ 


\title{
Maximal Regularity and Asymptotic Behavior of Solutions for the Cahn-Hilliard Equation with Dynamic Boundary Conditions
}

\author{
Jan Prüss \\ Reinhard Racke
}

Songmu Zheng

$\begin{array}{ll}\text { Department of Mathematics } & \text { Department of Mathematics } \\ \text { and Computer Science } & \text { and Statistics } \\ \text { Martin-Luther-University } & \text { University of Konstanz } \\ \text { 60120 Halle } & 78457 \text { Konstanz } \\ \text { Germany } & \text { Germany } \\ \text { pruess@mathematik.uni-halle.de } & \text { reinhard.racke@uni-konstanz.de } \\ & \\ \text { Institute of Mathematics } \\ \text { Fudan University } \\ \text { Shanghai 200433 } \\ \text { P.R.China } \\ \text { szheng@fudan.ac.cn }\end{array}$

\begin{abstract}
This paper deals with the Cahn-Hilliard equation

$$
\psi_{t}=\Delta \mu, \quad \mu=-\Delta \psi-\psi+\psi^{3}, \quad(t, x) \in J \times \Omega,
$$

subject to the boundary conditions

$$
\frac{1}{\Gamma_{s}} \psi_{t}=\sigma_{s} \Delta_{||} \psi-\partial_{\nu} \psi-g_{s} \psi+h, \quad \partial_{\nu} \mu=0,
$$

and the initial condition $\psi(0, x)=\psi_{0}(x)$ where $J=(0, \infty)$, and $\Omega \subset \mathbb{R}^{n}$ is a bounded domain with smooth boundary $\Gamma=\partial G, n \leq 3$, and $\Gamma_{s}, \sigma_{s}, g_{s}>0, h$ are constants.

This problem has already been considered in the recent paper of Racke and Zheng [13] where global existence and uniqueness were obtained. In this paper we first obtain the results on maximal $L_{p}$-regularity of solution and prove that the solution defines a $C_{0}$-semigroup in certain Sobolev spaces. We then study the asymptotic behavior of the solutions of this problem and prove the existence of global attractors.
\end{abstract}




\section{Introduction}

Let $\Omega \subset \mathbb{R}^{n}$ be a bounded domain with boundary $\Gamma=\partial \Omega$ of class $C^{4}$, and consider the following boundary value problem for the Cahn-Hilliard equation.

$$
\begin{array}{r}
\psi_{t}=\Delta \mu, \quad \mu=-\Delta \psi-\psi+\psi^{3}, \quad t>0, x \in \Omega, \\
\partial_{\nu} \mu=0, \quad t>0, x \in \Gamma \\
\partial_{t} \psi-\sigma_{s} \Delta_{\|} u+\partial_{\nu} \psi+g_{s} \psi=h, \quad t>0, x \in \Gamma \\
\psi=\psi_{0} \quad t=0, x \in \Omega .
\end{array}
$$

Here $\nu(x)$ denotes the outer normal of $\Omega$ at $x \in \Gamma, \Delta_{\|}$means the Laplace-Beltrami operator on $\Gamma$, and $\Gamma_{s}, \sigma_{s}, g_{s}>0, h$ are constants. This problem arises from the study of spinodal decomposition of binary mixtures that appears, for examples, in cooling processes of alloys, glasses or polymer mixtures (see Cahn and Hilliard [2], Novick-Cohen and Segal [10], Kenzler et al. [8], and the references cited therein.) Boundary condition (1.3) is usually called the dynamic boundary condition since it also involves the time derivative of dependent function $\psi$. It is derived when the effective interaction between the wall (i.e., the boundary $\Gamma$ ) and two mixture components are short-ranged (see Kenzler et al. [8]).

This problem was recently studied by Racke and Zheng [13] and the global existence and uniqueness of solution was proved there. Furthermore, it was pointed out that for $t>0$ the solution is $C^{\infty}$. However, it was not clear whether the solution defines a $C_{0}$-semigroup in the Sobolev space $V$ introduced in that paper. In the present paper we further investigate this problem. More precisely, we prove maximal $L_{p}$-regularity of solution which implies that the solution defines a $C_{0}$-semigroup in certain Sobolev spaces. Furthermore, we prove the existence of a global attractor for this problem.

This paper is organized as follows. In Section 2, we first study a linear problem associated with our original problem (1.1)-(1.4), and we establish maximal $L_{p}$-regularity results. Then we consider the corresponding nonlinear problem (1.1)-(1.4), and prove in Sections 3 and 4 local well-posedness as well as global well-posedness in a different phase manifold $\mathcal{M}$ than $V$, which implies that the solution defines a $C_{0}$-semigroup in $\mathcal{M}$. In the final section we prove the existence of a global attractor in $M_{2}$ (for $p=2$ ) as well as in $V$.

\section{The Linear Problem}

In this section we study the following linearized version of (1.1).

$$
\begin{aligned}
\partial_{t} v+\Delta^{2} v & =f, \quad t>0, x \in \Omega, \\
\partial_{\nu} \Delta v & =g, \quad t>0, x \in \Gamma \\
\frac{1}{\Gamma_{s}} \partial_{t} v-\sigma_{s} \Delta_{\|} v+\gamma \partial_{\nu} v+g_{s} v & =h, \quad t>0, x \in \Gamma \\
v & =v_{0} \quad t=0, x \in \Omega .
\end{aligned}
$$

Here the functions $f, g, h$ as well as the initial value $v_{0}$ are given; $\sigma_{s}, \gamma>0$, and $g_{s} \geq 0$ are given constants ( $\gamma=1$ in the original system). 
Let $J=[0, T]$ and $1<p<\infty$. We are looking for solutions in the class

$$
v \in H_{p}^{1}\left(J ; L_{p}(\Omega)\right) \cap L_{p}\left(J ; H_{p}^{4}(\Omega)\right),
$$

which is the natural class for $(2.1)$ in the $L_{p}$-setting. Then by well-known trace theorems (cf. [9], [1], [4]) the data $f, g, v_{0}$ necessarily satisfy

$$
f \in L_{p}(J \times \Omega), \quad g \in W_{p}^{1 / 4-1 / 4 p}\left(J ; L_{p}(\Gamma)\right) \cap L_{p}\left(J ; W_{p}^{1-1 / p}(\Gamma)\right), \quad v_{0} \in W_{p}^{4-4 / p}(\Omega) .
$$

As usual, here and in the sequel $W_{p}^{s}$ denote the fractional Sobolev spaces. Furthermore, the traces of $v$ and $\partial_{\nu} v$ on $\Gamma$ satisfy

$$
\left.v\right|_{\Gamma} \in W_{p}^{1-1 / 4 p}\left(J ; L_{p}(\Gamma)\right) \cap L_{p}\left(J ; W_{p}^{4-1 / p}(\Gamma)\right),
$$

and

$$
\left.\partial_{\nu} v\right|_{\Gamma} \in W_{p}^{3 / 4-1 / 4 p}\left(J ; L_{p}(\Gamma)\right) \cap L_{p}\left(J ; W_{p}^{3-1 / p}(\Gamma)\right) .
$$

This leaves some choice for the setting of the dynamic boundary condition. The possibility of lowest order is the choice $h \in L_{p}\left(J ; W_{p}^{2-1 / p}(\Omega)\right)$. Looking at the dynamical boundary condition as a heat equation on $J \times \Gamma$, this will result in

$$
\left.v\right|_{\Gamma} \in H_{p}^{1}\left(J ; W_{p}^{2-1 / p}(\Gamma)\right) \cap L_{p}\left(J ; W_{p}^{4-1 / p}(\Gamma)\right) .
$$

This regularity implies that the trace of $\left.v\right|_{\Gamma}$ at $t=0$ necessarily satisfies $\left.v_{0}\right|_{\Gamma} \in W_{p}^{4-3 / p}(\Gamma)$.

The other extreme possibility consists in taking the regularity of the normal derivative of $v$ as the basic regularity, i.e. we may consider the class

$$
h \in W_{p}^{3 / 4-1 / 4 p}\left(J ; L_{p}(\Gamma)\right) \cap L_{p}\left(J ; W_{p}^{3-1 / p}(\Gamma)\right) .
$$

This leads to

$$
\left.v\right|_{\Gamma} \in W_{p}^{7 / 4-1 / 4 p}\left(J ; L_{p}(\Gamma)\right) \cap H_{p}^{1}\left(J ; W_{p}^{3-1 / p}(\Gamma)\right) \cap L_{p}\left(J ; W_{p}^{5-1 / p}(\Gamma)\right) .
$$

Then necessarily $\left.v_{0}\right|_{\Gamma} \in W_{p}^{5-3 / p}(\Gamma)$ and the compatibility conditions

$$
\left.\partial_{\nu} \Delta v_{0}\right|_{\Gamma}=\left.g\right|_{t=0}, \quad \text { for } p>5,
$$

and

$$
\left.\sigma_{s} \Delta_{||} v_{0}\right|_{\Gamma}-\left.\gamma \partial_{\nu} v_{0}\right|_{\Gamma}-\left.g_{s} v_{0}\right|_{\Gamma}+\left.h\right|_{t=0} \in W_{p}^{3-5 / p}(\Gamma), \quad \text { for } p>5 / 3,
$$

must hold.

More generally, any choice of the space for $h$ of the type

$$
h \in W_{p}^{s}\left(J ; L_{p}(\Gamma)\right) \cap L_{p}\left(J ; W_{p}^{r}(\Gamma)\right), \quad 0 \leq s \leq 3 / 4-1 / 4 p, 2-1 / p \leq r \leq 3-1 / p,
$$

will work. Observe that for such $r, s$ the inequality $2 s \leq r$ is valid. The corresponding trace space for $\left.v_{0}\right|_{\Gamma}$ now becomes $W_{p}^{r+2-2 / p}(\Gamma)$, and in case $s>1 / p$ also the time derivative $\left.\partial_{t} v\right|_{\Gamma}$ has trace at $t=0$, which belongs to $W_{p}^{r(1-1 / s p)}(\Gamma)$.

Here is the main result on maximal $L_{p}$-regularity of (2.1). 
Theorem 2.1. Let $n \in \mathbb{N}, 1<p<\infty$, fix numbers $s \in[0,3 / 4-1 / 4 p]$ and $r \in$ $[2-1 / p, 3-1 / p], p \neq 5$, sp $\neq 1$, and let $\sigma_{s}, \gamma>0$. Suppose $\Omega \subset \mathbb{R}^{n}$ is bounded with boundary $\Gamma=\partial \Omega \in C^{r+2}$, and let $J=[0, T]$.

Then there is a unique solution $v$ of (2.1) such that

$$
v \in Z:=H_{p}^{1}\left(J ; L_{p}(\Omega)\right) \cap L_{p}\left(J ; H_{p}^{4}(\Omega)\right),
$$

and

$$
\left.v\right|_{\Gamma} \in Z_{\Gamma}:=W_{p}^{s+1}\left(J ; L_{p}(\Gamma)\right) \cap H_{p}^{1}\left(J ; W_{p}^{r}(\Gamma)\right) \cap L_{p}\left(J ; W_{p}^{r+2}(\Gamma)\right),
$$

if and only if the data are subject to the following conditions.

(a) $f \in X:=L_{p}(J \times \Omega), g \in Y_{\Gamma}:=W_{p}^{1 / 4-1 / 4 p}\left(J ; L_{p}(\Gamma)\right) \cap L_{p}\left(J ; W_{p}^{1-1 / p}(\Gamma)\right)$,

$v_{0} \in X_{p}:=\left\{v \in W_{p}^{4-4 / p}(\Omega):\left.v\right|_{\Gamma} \in W_{p}^{r+2-2 / p}(\Gamma)\right\}$;

(b) $h \in X_{\Gamma}:=W_{p}^{s}\left(J ; L_{p}(\Gamma)\right) \cap L_{p}\left(J ; W_{p}^{r}(\Gamma)\right)$,

(c) $\left.\partial_{\nu} \Delta v_{0}\right|_{\Gamma}=\left.g\right|_{t=0}$, if $p>5$;

(d) $v_{1}:=\left.\sigma_{s} \Delta_{||} v_{0}\right|_{\Gamma}-\left.\gamma \partial_{\nu} v_{0}\right|_{\Gamma}-\left.g_{s} v_{0}\right|_{\Gamma}+\left.h\right|_{t=0} \in W_{p}^{r(1-1 / p s)}(\Gamma)$, if $s>1 / p$.

Proof. Necessity of $(\mathrm{a}) \sim(\mathrm{d})$ is a consequence of well-known trace theorems and has been already explained. The values $p=5$ and $s p=1$ have been excluded since the trace theorems leading to (c) and (d) are not true for these values of $p$.

For the sufficiency part, the main idea is to reduce the problem to an equation for $\bar{\rho}:=\left.u\right|_{\Gamma}$ on $J \times \Gamma$. This will be done as follows. Let $A:=-\sigma_{s} \Delta_{\|}$denote the LaplaceBeltrami on $W_{p}^{r}(\Gamma)$. It is well-known that this operator is the negative generator of an analytic $C_{0}$-semigroup which enjoys maximal $L_{p}$-regularity. First, assuming that $\left.u\right|_{\Gamma}$ is known, it is convenient to decompose $\bar{\rho}=\left.u\right|_{\Gamma}$ as

$$
\left.u\right|_{\Gamma}=\bar{\rho}=\rho+\rho_{1},
$$

where

$$
\rho_{1}=\left.e^{-A t} v_{0}\right|_{\Gamma}, \quad t>0
$$

in case $s p<1$, and

$$
\rho_{1}=e^{-A t}\left(\left.v_{0}\right|_{\Gamma}-\left.t A v_{0}\right|_{\Gamma}\right)+t e^{-A^{\alpha} t} v_{1}, \quad t>0,
$$

if $s p>1$; here we have set $\alpha=r / 2 s$. Then, by (b) and (c) the function $\rho_{1}$ belongs to $Z_{\Gamma}$, as elementary properties of analytic semigroups and their trace spaces show; cf. e.g. Prüss [12], where the necessary arguments are given.

Next we employ a recent result due to Denk, Hieber and Prüss [4] concerning the solvability of general parabolic initial boundary value problems, which means that for given $f, g, v_{0}$ satisfying (a) and (c), and $\rho_{1} \in W_{p}^{1-1 / 4 p}\left(J ; L_{p}(\Gamma)\right) \cap L_{p}\left(J ; W_{p}^{4-1 / p}(\Gamma)\right)$ defined as above, there is precisely one solution $v_{1} \in H_{p}^{1}\left(J ; L_{p}(\Omega)\right) \cap L_{p}\left(J ; H_{p}^{4}(\Omega)\right)$ of the problem

$$
\begin{aligned}
& \partial_{t} v+\Delta^{2} v=f, \quad t \in J, x \in \Omega, \\
& \partial_{\nu} \Delta v=g,\left.\quad v\right|_{\Gamma}=\rho_{1}, \quad t \in J, x \in \Gamma, \\
& \left.v\right|_{t=0}=v_{0}, \quad x \in \Omega .
\end{aligned}
$$


Thus the function $v_{1}$ belongs to $Z$ and is determined only by the data $f, g$, and $v_{0}$, and $\partial_{\nu} v_{1}$ belongs to $X_{\Gamma}$, taking traces.

Next we derive the governing equation for the linear problem (2.1), i.e. the equation for $\rho$. For this purpose we let the solution of

$$
\begin{aligned}
& \partial_{t} v+\Delta^{2} v=0, \quad t \in J, x \in \Omega, \\
& \partial_{\nu} \Delta v=0,\left.\quad v\right|_{\Gamma}=\rho, \quad t \in J, x \in \Gamma, \\
& \left.v\right|_{t=0}=0, \quad x \in \Omega,
\end{aligned}
$$

be denoted by $R \rho$. Note that in case

$$
\rho \in{ }_{0} W_{p}^{1-1 / 4 p}\left(J ; L_{p}(\Gamma)\right) \cap L_{p}\left(J ; W_{p}^{4-1 / p}(\Gamma)\right),
$$

then

$$
R \rho \in{ }_{0} Z:={ }_{0} H_{p}^{1}\left(J ; L_{p}(\Omega)\right) \cap L_{p}\left(J ; H_{p}^{2}(\Omega)\right) .
$$

Here, and in the following the left subscript 0 means that all existing traces at $t=0$ vanish. Thus $v=v_{1}+R \rho$ solves the problem

$$
\begin{aligned}
& \partial_{t} v+\Delta^{2} v=f, \quad t \in J, x \in \Omega, \\
& \partial_{\nu} \Delta v=g,\left.\quad v\right|_{\Gamma}=\rho+\rho_{1}, \quad t \in J, x \in \Gamma, \\
& \left.v\right|_{t=0}=v_{0}, \quad x \in \Omega .
\end{aligned}
$$

Inserting $v$ into the dynamic boundary condition we obtain the following problem for $\rho$.

$$
\frac{1}{\Gamma_{s}} \partial_{t} \rho-\sigma_{s} \Delta_{\|} \rho+\gamma \partial_{\nu} R \rho+g_{s} \rho=h_{1}, \quad t>0, x \in \Gamma, \rho(0)=0,
$$

where $h_{1}$ given by

$$
h_{1}=h-\left(\frac{1}{\Gamma_{s}} \partial_{t}-\sigma_{s} \Delta_{\|}\right) \rho_{1}-\gamma \partial_{\nu} v_{1}-g_{s} \rho_{1}
$$

belongs to

$$
{ }_{0} Z_{\Gamma}={ }_{0} W_{p}^{s}\left(J ; L_{p}(\Gamma)\right) \cap L_{p}\left(J ; W_{p}^{r}(\Gamma)\right),
$$

by construction; note that $s \leq 3 / 4-1 / 4 p$ and $r \leq 3-1 / p$. Thus (2.1) is reduced to problem (2.4) for $\rho$.

Introducing the operators $L:=\frac{1}{\Gamma_{s}} \partial_{t}-\sigma_{s} \Delta_{\|}+g_{s} I$ and $S \rho:=\partial_{\nu} R \rho$, we may reformulate the latter problem as an operator equation

$$
L \rho+\gamma S \rho=h_{1} .
$$

Since $A=-\sigma_{s} \Delta_{\|}$has maximal $L_{p}$-regularity, $L:{ }_{0} Z_{\Gamma} \rightarrow{ }_{0} X_{\Gamma}$ is an isomorphism. Let us consider $S$ in more detail. For obvious reasons, we would call $S$ the parabolic DirichletNeumann operator. It has order $1 / 4$ in time and order 1 in space. Therefore, it is subordinate to $L$. Moreover, it is causal, i.e. $S$ is an integro-differential operator of Volterra type of inhomogeneous fractional order, and it is of order 1 and nonlocal in the variables $x \in \Gamma$. 
Now we are going to prove that $L+\gamma S$ is an isomorphism for each $\gamma>0$. First we show injectivity. Suppose that $L \rho+\gamma S \rho=0$; let $u$ denote the solution of

$$
\begin{aligned}
& \partial_{t} u+\Delta^{2} u=0, \quad t>0, x \in \Omega, \\
& \partial_{\nu} \Delta u=0, \quad u=\rho, \quad t>0, x \in \Gamma, \\
& u=0, \quad t=0, x \in \Omega
\end{aligned}
$$

Then

$$
\frac{1}{2} \partial_{t}\left[\gamma|\nabla u|_{2}^{2}+\sigma_{s}|\nabla||\rho|_{2}^{2}+g_{s}|\rho|^{2}\right]=-\gamma|\nabla \Delta u|_{2}^{2}-\frac{1}{\Gamma_{s}}\left|\partial_{t} \rho\right|_{2}^{2} \leq 0
$$

which implies $u=\rho=0$, i.e. $L+\gamma S$ is injective. Next we show that the range of $L+\gamma S$ is closed. If it is not true, then there is a sequence $\left(\rho_{n}\right) \subset Z_{\Gamma}$ with $\left\|\rho_{n}\right\|_{Z_{\Gamma}}=1$ such that $h_{n}=(L+\gamma S) \rho_{n} \rightarrow 0$ in $X_{\Gamma}$. Thus

$$
\left(L^{-1} S \rho_{n}\right) \subset{ }_{0} W_{p}^{7 / 4-1 / 4 p}\left(J ; L_{p}(\Gamma)\right) \cap{ }_{0} H_{p}^{1}\left(J ; W_{p}^{3-1 / p}(\Gamma)\right) \cap L_{p}\left(J ; W_{p}^{5-1 / p}(\Gamma)\right)
$$

is bounded. Hence, by compact embedding it is relatively compact in ${ }_{0} W_{p}^{1-1 / 4 p}\left(J ; L_{p}(\Gamma)\right) \cap$ $L_{p}\left(J ; W_{p}^{4-1 / p}(\Gamma)\right)$, which implies that $\left(S \rho_{n}\right)$ has a convergent subsequence in $X_{\Gamma}$. Thus, this yields a convergent subsequence $\rho_{n_{k}} \rightarrow \rho_{0}$ in $Z_{\Gamma}$ because $L$ is an isomorphism. Since $S$ is bounded, we conclude that $(L+\gamma S) \rho_{0}=0$, i.e. $\rho_{0}=0$ by injectivity of $L+\gamma S$, a contradiction to $\left\|\rho_{0}\right\|_{Z_{\Gamma}}=1$. Therefore, $L+\gamma S$ is an injective Fredholm operator of index 0 , which means that it is an isomorphism for each $\gamma>0$. This completes the proof of Theorem 1.

Remark 2.2. There is an analytic $C_{0}$-semigroup hidden in Theorem 2.1. To see this consider the space $\mathbb{X}:=L_{p}(\Omega) \times W_{p}^{r}(\Gamma)$ and define an operator $\mathbb{A}$ by means of

$$
\mathbb{A}(v, \rho)=\left(\Delta^{2} v, \gamma \partial_{\nu} v-\sigma_{s} \Delta_{\|} \rho+g_{s} \rho\right),
$$

for $(v, \rho) \in D(\mathbb{A})$, where

$$
D(\mathbb{A})=\left\{(v, \rho) \in \mathbb{X}: v \in H_{p}^{4}(\Omega), \rho \in W_{p}^{r+2}(\Gamma),\left.v\right|_{\Gamma}=\rho,\left.\partial_{\nu} \Delta v\right|_{\Gamma}=0\right\} .
$$

Here as before $r \in[2-1 / p, 3-1 / p]$. This operator $\mathbb{A}$ is closed, linear and densely defined, and (2.1) with $g=0$ is equivalent to

$$
\dot{z}+\mathbb{A} z=F, \quad z(0)=z_{0},
$$

where $z=(v, \rho), F=(f, h)$ and $z_{0}=\left(v_{0},\left.v_{0}\right|_{\Gamma}\right)$. It is easy to see that $(2.5)$ has maximal $L_{p}$-regularity if and only if for each $f \in L_{p}(J \times \Omega)$ and $h \in L_{p}\left(J ; W_{p}^{r}(\Gamma)\right)$ there is a unique solution $z$ of (2.5) such that $v \in Z, \rho=v_{\Gamma} \in Z_{\Gamma}$ with $s=0$. Therefore, Theorem 2.1 implies that (2.5) has maximal $L_{p}$-regularity, and then by a result in Hieber and Prüss $[5],\left(\right.$ see also [11], [1]), $-\mathbb{A}$ generates an analytic $C_{0}$-semigroup in $\mathbb{X}$. 


\section{Local Well-Posedness}

In this section we want to solve the nonlinear problem, i.e.

$$
\begin{aligned}
\partial_{t} \psi+\Delta^{2} \psi-\Delta \psi & =\Delta \psi^{3}+f, \quad t \in J, x \in \Omega, \\
\partial_{\nu} \Delta \psi+\partial_{\nu} \psi & =\partial_{\nu} \psi^{3}+g, \quad t \in J, x \in \Gamma \\
\frac{1}{\Gamma_{s}} \partial_{t} \psi-\sigma_{s} \Delta_{||} \psi+\partial_{\nu} \psi+g_{s} \psi & =h, \quad t \in J, x \in \Gamma \\
\psi=\psi_{0} \quad t=0, x \in \Omega . &
\end{aligned}
$$

This problem has been solved in a recent paper Racke and Zheng [13]. In this section and the next section we would like to reconsider this problem in different spaces based on the $L_{p}$-regularity obtained in the previous section. We first want to employ the contraction mapping principle to solve this problem on a possibly smaller time interval $J_{a}=[0, a] \subset J$. For this purpose, let $\psi_{0} \in X_{p}, f \in X, g \in Y_{\Gamma}$ and $h \in X_{\Gamma}$ be given such that the compatibility conditions

$$
\begin{aligned}
& \left.\partial_{\nu} \Delta \psi_{0}\right|_{\Gamma}=\left.\partial_{\nu}\left(-\psi_{0}+\psi_{0}^{3}\right)\right|_{\Gamma}+\left.g\right|_{t=0}, \quad p>5 \\
& \left.\sigma_{s} \Delta|| \psi_{0}\right|_{\Gamma}-\left.\gamma \partial_{\nu} \psi_{0}\right|_{\Gamma}-\left.g_{s} \psi_{0}\right|_{\Gamma}+\left.h\right|_{t=0} \in W_{p}^{r(1-1 / p s)}(\Gamma), \quad s>1 / p
\end{aligned}
$$

are satisfied. Here we have used the notation of Section 2. In the sequel we denote the relevant spaces by $X(a), Y_{\Gamma}(a)$ to indicate the time interval under consideration, e.g. $J_{a}$, and so on.

The main result of this section reads as follows.

Theorem 3.1. Let $1<p<\infty, p \geq(n+4) / 5, s \in[0,3 / 4-1 / 4 p], r \in[2-1 / p, 3-1 / p]$, and let the data $\psi_{0} \in X_{p}, f \in X(T), g \in Y_{\Gamma}(T)$, and $h \in X_{\Gamma}(T)$ be given such that the compatibility conditions (3.2) are satisfied. Then there is $a \in(0, T]$ and a unique solution of (3.1) in the class $\psi \in Z(a)$. Furthermore, $\psi_{\Gamma} \in Z_{\Gamma}(a)$. $\psi$ depends continuously on the data, and for $f, g, h$ independent of $t$, the map $\psi_{0} \mapsto \psi(t)$ defines a local semiflow in the natural phase manifold $\mathcal{M}$ defined by $X_{p}$ and the compatibility conditions (3.2).

Proof. Thanks to Theorem 2.1, we may define a function $u^{*} \in Z(T)$ with $\left.u^{*}\right|_{\Gamma} \in Z_{\Gamma}(T)$ as the solution of the special problem

$$
\begin{aligned}
& \partial_{t} u^{*}+\Delta^{2} u^{*}=f, \quad t \in J, x \in \Omega, \\
& \partial_{\nu} \Delta u^{*}=g-e^{-A^{2} t} g_{0}, \quad t \in J, x \in \Gamma, \\
& \partial_{t} u^{*}-\sigma_{s} \Delta_{\|} u^{*}+\gamma \partial_{\nu} u^{*}+g_{s} u^{*}=h, \quad t \in J, x \in \Gamma, \\
& \left.u^{*}\right|_{t=0}=\psi_{0}, \quad x \in \Omega,
\end{aligned}
$$

where as before $A=-\Delta_{\|}$. In case $p \leq 5$, we set $g_{0}=0$, and $g_{0}=\left.g\right|_{t=0}-\left.\partial_{\nu} \Delta \psi_{0}\right|_{\Gamma}$ if $p>5$. For a given $a \in(0, T]$ to be fixed later, we define

$$
\mathbb{E}_{1}:=\left\{u \in Z(a):\left.u\right|_{\Gamma} \in Z_{\Gamma}(a)\right\}, \quad \text { and }{ }_{0} \mathbb{E}_{1}:=\left\{u \in \mathbb{E}_{1}:\left.u\right|_{t=0}=0\right\}
$$

with canonical norm $\|\cdot\|_{1}$ and

$$
\mathbb{E}_{0}:=X(a) \times Y_{\Gamma}(a) \times X_{\Gamma}(a), \quad \text { and }{ }_{0} \mathbb{E}_{0}:=\left\{(f, g, h) \in \mathbb{E}_{0}:\left.g\right|_{t=0}=\left.h\right|_{t=0}=0\right\}
$$


with norm $\|\cdot\|_{0}$. Define the linear operator $\mathbb{L}: \mathbb{E}_{1} \rightarrow \mathbb{E}_{0}$ by means of

$$
\mathbb{L} v=\left[\begin{array}{l}
\partial_{t} v+\Delta^{2} v \\
\partial_{\nu} \Delta v \\
\left.\partial_{t} v\right|_{\Gamma}-\left.\sigma_{s} \Delta|| v\right|_{\Gamma}+\left.\partial_{\nu} v\right|_{\Gamma}+\left.g_{s} v\right|_{\Gamma}
\end{array}\right] .
$$

By Theorem 2.1, $\mathbb{L}:{ }_{0} \mathbb{E}_{1} \rightarrow{ }_{0} \mathbb{E}_{0}$ is linear, bounded and bijective, hence an isomorphism. Next we define the nonlinear mapping $F: \mathbb{E}_{1} \times{ }_{0} \mathbb{E}_{1} \rightarrow{ }_{0} \mathbb{E}_{0}$ by

$$
F\left(u^{*}, v\right)=\left[\begin{array}{l}
\Delta\left(-\left(u^{*}+v\right)+\left(u^{*}+v\right)^{3}\right) \\
\partial_{\nu}\left(-\left(u^{*}+v\right)+\left(u^{*}+v\right)^{3}\right)-g_{1} \\
0
\end{array}\right]
$$

where $g_{1}(t)=e^{-A^{2} t}\left[\left.\partial_{\nu}\left(-u^{*}+\left(u^{*}\right)^{3}\right)\right|_{t=0}\right] \in Y_{\Gamma}$. Then $u=u^{*}+v$ is a solution of (3.1) if and only if

$$
\mathbb{L} v=F\left(u^{*}, v\right) \quad \text { i.e. } \quad v=\mathbb{L}^{-1} F\left(u^{*}, v\right) .
$$

To show that $F: \mathbb{E}_{1} \times{ }_{0} \mathbb{E}_{1} \rightarrow{ }_{0} \mathbb{E}_{0}$ is of class $C^{1}$, it is sufficient to show that the cubic form $u \mapsto u^{3}$ is well defined and bounded from $\mathbb{E}_{1}$ to $H_{2}^{1 / 2}\left(J ; L_{p}(\Omega)\right) \cap L_{p}\left(J ; H_{p}^{2}(\Omega)\right)$. In fact, if the latter is valid, then $u \mapsto \Delta u^{3}$ is bounded from $\mathbb{E}_{1}$ to $X$, and after taking traces, the boundedness of $u \mapsto \partial_{\nu} u^{3}$ from $\mathbb{E}_{1}$ to $Y_{\Gamma}$ follows as well. These properties then show that $F$ is even real analytic in $\left(u^{*}, v\right)$. The following lemma takes care of the cubic form.

Lemma 3.2. Let $p \geq(n+4) / 5$. Then the trilinear form $b(u, v, w):=u v w$ is bounded from $\mathbb{E}_{1}$ to $H_{2}^{1 / 2}\left(J ; L_{p}(\Omega)\right) \cap L_{p}\left(J ; H_{p}^{2}(\Omega)\right)$, and there is a constant $C>0$ such that the estimate

$$
\|u v w\|_{H_{p}^{1 / 2}\left(L_{p}\right) \cap L_{p}\left(H_{p}^{2}\right)} \leq C\|u\|_{Z}\|v\|_{Z}\|w\|_{Z}, \quad u, v, w \in Z
$$

is valid.

Proof. To prove boundedness in $L_{p}\left(J ; H_{p}^{2}(\Omega)\right)$, by symmetry we have to estimate the two typical terms like $\left(\partial_{i} \partial_{j} u\right) v w$ and $\left(\partial_{i} u\right)\left(\partial_{j} v\right) w$. The first one is estimated via Hölder's inequality like

$$
\left\|\left(\partial_{i} \partial_{j} u\right) v w\right\|_{p} \leq\left\|\left.\left(\partial_{i} \partial_{j} u\right)\right|_{5 p / 3}\right\| v\left\|_{5 p}\right\| w \|_{5 p} .
$$

The mixed derivative theorem and the Sobolev embedding theorem yields

$$
Z \hookrightarrow H_{p}^{1-\theta}\left(J ; H_{p}^{4 \theta}(\Omega)\right) \hookrightarrow L_{5 p}(J \times \Omega),
$$

provided $1-\theta-1 / p \geq-1 / 5 p$ and $4 \theta-n / p \geq-n / 5 p$, i.e. provided $n / 5 p \leq \theta \leq 1-4 / 5 p$ which is possible since $p \geq(n+4) / 5$. On the other hand,

$$
Z \hookrightarrow H_{p}^{1-\theta}\left(J ; H_{p}^{4 \theta}(\Omega)\right) \hookrightarrow L_{5 p / 3}\left(J ; H_{5 p / 3}^{2}(\Omega)\right),
$$

provided $1-\theta-1 / p \geq-3 / 5 p$ and $4 \theta-n / p \geq 2-3 n / 5 p$, i.e. provided $(1+n / 5 p) / 2 \leq$ $\theta \leq 1-2 / 5 p$ which again is possible for $p \geq(n+4) / 5$.

In a similar way the second type of terms $\left(\partial_{i} u\right)\left(\partial_{j} v\right) w$ can be treated, obtaining

$$
\left\|\left(\partial_{i} u\right)\left(\partial_{j} v\right) w\right\|_{p} \leq\left\|\partial_{i} u\right\|_{5 p / 2}\left\|\partial_{j} v\right\|_{5 p / 2}\|w\|_{5 p}
$$


To estimate $u v w$ in $H_{p}^{1 / 2}\left(J ; L_{p}(\Omega)\right)$, we employ the embedding theorem

$$
Z \hookrightarrow H_{p}^{1-\theta}\left(J ; H_{p}^{4 \theta}(\Omega)\right) \hookrightarrow H_{5 p / 3}^{1 / 2}\left(J ; L_{5 p / 3}(\Omega)\right),
$$

provided $1-\theta-1 / p \geq 1 / 2-3 / 5 p$ and $4 \theta-n / p \geq-3 n / 5 p$, i.e. provided $n / 10 p \leq \theta \leq$ $1 / 2-2 / 5 p$ which is again possible since $p \geq(n+4) / 5$.

We note further that we have also shown the estimate

$$
\|u\|_{H_{5 p / 3}^{1 / 2}\left(L_{5 p / 3}\right) \cap L_{5 p / 3}\left(H_{5 p / 3}^{2}\right)} \leq C\|u\|_{Z},
$$

where $C>0$ is independent of the interval under consideration.

Hence,

$$
\|\Delta u\|_{X(a)} \leq C a^{2 p / 5}\|u\|_{Z(a)}, \quad\left\|\partial_{\nu} u\right\|_{Y_{\Gamma}(a)} \leq C a^{2 p / 5}\|u\|_{Z(a)},
$$

on $J_{a}=[0, a]$.

Consider a ball $\mathbb{B}_{r}(0) \subset{ }_{0} \mathbb{E}_{1}$, where $r>0$ will be fixed later, and define $T: \mathbb{B}_{r}(0) \rightarrow_{0} \mathbb{E}_{1}$ by means of $T v:=\mathbb{L}^{-1} F\left(u^{*}, v\right)$. To show that $T$ is a contraction, we infer from Lemma 2.2 and (3.7) that

$$
\begin{aligned}
\|T v-T w\|_{1} & \leq\left\|\mathbb{L}^{-1}\right\|\left\|F\left(u^{*}, v\right)-F\left(u^{*}, w\right)\right\|_{0} \\
& \leq C\left[\|v-w\|_{H_{p}^{1 / 2}\left(L_{p}\right) \cap L_{p}\left(H_{p}^{2}\right)}+\|v-w\|_{1}\left(\|v\|_{1}+\|w\|_{1}\right)^{2}\right] \\
& \leq C\left[a^{2 p / 5}+4 r^{2}\right]\|v-w\|_{1} \\
& \leq \frac{1}{2}\|v-w\|_{1}, \quad v, w \in \mathbb{B}_{r}(0),
\end{aligned}
$$

provided $C\left[a^{2 p / 5}+4 r^{2}\right] \leq 1 / 2$, i.e. if $r>0$ and $a>0$ are sufficiently small.

To show that $T \mathbb{B}_{r}(0) \subset \mathbb{B}_{r}(0)$, in a similar way we obtain that

$$
\begin{aligned}
\|T v\|_{1} & \leq\left\|\mathbb{L}^{-1}\right\|\left\|F\left(u^{*}, v\right)\right\|_{0} \\
& \leq C\left[\left\|u^{*}+v\right\|_{H_{p}^{1 / 2}\left(L_{p}\right) \cap L_{p}\left(H_{p}^{2}\right)}+\left\|u^{*}+v\right\|_{1}^{3}+\left\|g_{0}\right\|_{Y_{\Gamma}(a)}\right] \\
& \leq C\left[\left\|u^{*}\right\|_{1}+a^{2 p / 5} r+\left(\left\|u^{*}\right\|_{1}+r\right)^{3}+\left\|g_{0}\right\|_{Y_{\Gamma}(a)} \leq r\right.
\end{aligned}
$$

provided $a>0$ and $r>0$ are small enough. Note that $\left\|u^{*}\right\|_{1} \rightarrow 0$ and also $\left\|g_{0}\right\|_{Y_{\Gamma}(a)} \rightarrow 0$ as $a \rightarrow 0$, since $u^{*}$ is a fixed function.

Therefore the contraction mapping principle yields a unique fixed point $v \in \mathbb{B}_{r}(0) \subset$ ${ }_{0} \mathbb{E}_{1}$ which in turn gives the unique solution $u=v+u^{*}$ of $(3.1)$. It is clear that $u^{*}$ depends continuously on the data and $v$ is continuous in $u^{*}$, hence $u$ depends continuously on the data as well. If $f, g, h$ are constant in $t$, problem (3.1) is autonomous, hence translation is invariant, which by uniqueness of solution shows that the map $u_{0} \mapsto u(t)$ is a local semiflow in the phase space $X_{p}$. This completes the proof.

Successive application of Theorem 3.1 yields a solution $u$ on a maximal time interval $J_{\max }=\left[0, a_{\max }\right)$. This time interval is characterized by the two equivalent conditions

$$
\lim _{t \rightarrow t_{\max }} \psi(t) \quad \text { does not exist in } X_{p}
$$


and

$$
\|\psi\|_{Z\left(a_{\max }\right)}+\left\|\left.\psi\right|_{\Gamma}\right\|_{Z_{\Gamma}\left(a_{\max }\right)}=\infty .
$$

Moreover, the function $F$ defined above is real analytic, and $D_{v} F\left(u^{*}, v\right)$ is of lower order, compared to $\mathbb{L}$. Therefore, by arguments as in the proof of Theorem 3.1 one can show that $\mathbb{L}-D_{v} F\left(u^{*}, v\right):{ }_{0} \mathbb{E}_{1} \rightarrow{ }_{0} \mathbb{E}_{0}$ is an isomorhism for each $u^{*}$ and $v$. Therefore the solution $\psi$ depends on the data even analytically.

\section{Global Well-Posedness}

In this section we want to solve the nonlinear problem

$$
\begin{array}{r}
\partial_{t} \psi+\Delta^{2} \psi+\Delta \psi=\Delta \psi^{3}+f, \quad t \in J, x \in \Omega, \\
\partial_{\nu} \Delta \psi+\partial_{\nu} \psi=\partial_{\nu} \psi^{3}+g, \quad t \in J, x \in \Gamma \\
\frac{1}{\Gamma_{s}} \partial_{t} \psi-\sigma_{s} \Delta \|_{\|} \psi+\partial_{\nu} \psi+g_{s} \psi=h, \quad t \in J, x \in \Gamma \\
\psi=\psi_{0} \quad t=0, x \in \Omega .
\end{array}
$$

globally in time in the setting established in previous sections. In particular, we would like to show that the solution defines a semiflow in suitable phase space $\mathcal{M}$. Here we finally have to restrict to dimensions $n<4$. For this purpose let data $\psi_{0} \in X_{p}, f \in X(T), g \in Y_{\Gamma}(T)$ and $h \in X_{\Gamma}(T)$ be given such that the compatibility conditions (3.2) are satisfied. By Theorem 3.1 there is a unique solution on some maximal time interval $J_{\max }=\left[0, a_{\max }\right)$. We fix some arbitrary $0<a<a_{\max }$.

To prove the global existence, it is crucial to use the following well-known energy functional (cf. e.g. Racke and Zheng [13]):

$$
F[\psi]:=\frac{1}{2} \int_{\Omega}\left[|\nabla \psi|^{2}-|\psi|^{2}+\frac{1}{2}|\psi|^{4}\right] d x+\frac{1}{2} \int_{\Gamma}\left[\sigma_{s}\left|\nabla_{\|} \psi\right|^{2}+g_{s}|\psi|^{2}\right] d s,
$$

where $d s$ means the surface measure on $\Gamma$ and $\nabla_{\|}$is the surface gradient. Computing the time derivative of $\Phi(u(t))$, we obtain by integration by parts, employing the boundary condition and using $\mu=-\Delta \psi-\psi+\psi^{3}$

$$
\begin{aligned}
& \frac{d}{d t} F[\psi(t)]=\int_{\Omega}\left[\partial_{t} \nabla \psi \cdot \nabla \psi-\psi \partial_{t} \psi+\psi^{3} \partial_{t} \psi\right] d x+\int_{\Gamma}\left[\sigma_{s} \partial_{t} \nabla_{\|} \psi \nabla_{\|} \psi+g_{s} \psi \partial_{t} \psi\right] d s \\
& =\int_{\Omega} \partial_{t} \psi\left[-\Delta \psi-\psi+\psi^{3}\right] d x+\int_{\Gamma} \partial_{t} \psi\left[\partial_{\nu} \psi-\sigma_{s} \Delta \| \psi+g_{s} \psi\right] d s \\
& =\int_{\Omega}(\Delta \mu+f) \mu d x+\int_{\Gamma} \partial_{t} \psi\left(h-\partial_{t} \psi\right) d s \\
& =-\int_{\Omega}\left[|\nabla \mu|^{2}-\mu f\right] d x-\int_{\Gamma}\left[\left|\partial_{t} \psi\right|^{2}-\partial_{t} \psi h-\mu g\right] d s, \\
& \leq-\int_{\Omega}|\nabla \mu|^{2} d x-\frac{3}{4} \int_{\Gamma}\left|\partial_{t} \psi\right|^{2} d s+\frac{1}{4} \int_{\Gamma}|h|^{2} d s+\left[\int_{\Omega} \mu f d x+\int_{\Gamma} \mu g d s\right] .
\end{aligned}
$$

In the last step we have used Young's inequality. The embedding $H_{2}^{1}(\Omega) \hookrightarrow L_{2}(\Gamma)$, and Poincaré's second inequality

$$
\int_{\Omega}|\psi|^{2} d y \leq C\left(\int_{\Omega}|\nabla \psi|^{2} d x+\left|\int_{\Omega} \psi d x\right|^{2}\right)
$$


imply further

$$
\begin{aligned}
& \left|\int_{\Omega} \mu f d x\right| \leq C|f|_{2}\left[|\nabla \mu|_{2}+\left|\int_{\Omega} \mu d x\right|\right] \\
& \left|\int_{\Gamma} \mu g\right| \leq C|g|_{2, \Gamma}\left[|\nabla \mu|_{2}+\left|\int_{\Omega} \mu d x\right|\right]
\end{aligned}
$$

where $|\cdot|_{p}$ and $|\cdot|_{p, \Gamma}$ denote the $L_{p}$-norms on $\Omega$ and on. $\Gamma$, respectively. Since

$$
\int_{\Omega} \mu d x=\int_{\Omega}\left(\psi^{3}-\psi\right) d x+\int_{\Gamma}\left(-h+\partial_{t} \psi+g_{s} \psi\right) d s
$$

we obtain

$$
\left|\int_{\Omega} \mu d x\right| \leq C\left[|\psi|_{4}^{3 / 4}+|h|_{2, \Gamma}+\left|\partial_{t} \psi\right|_{2, \Gamma}+|\psi|_{2, \Gamma}\right]
$$

Combining these estimates, we deduce from Young's inequality that

$$
\frac{d}{d t} F[\psi(t)] \leq-\frac{1}{2}\left[|\nabla \mu|_{2}^{2}+\left|\partial_{t} \psi\right|_{2, \Gamma}^{2}\right]+C\left[|\psi|_{4}^{4}+|\psi|_{2, \Gamma}^{2}+|f|_{2}^{4}+|g|_{2, \Gamma}^{4}+|h|_{2, \Gamma}^{2}\right] .
$$

By Gronwall's inequality, this implies the following a priori estimates.

Lemma 4.1. Let $p \geq 2$ and suppose $\psi$ is a solution of (4.1) on the maximal interval $J_{\max }$. Then there is a constant $C>0$ depending only on $T>0, \Omega$, and on the parameters $g_{s}$ and $\sigma_{s}$ such that

$$
\begin{aligned}
& \sup _{t \in J_{\text {max }}}\left[|\psi(t)|_{H_{2}^{1}(\Omega)}+|\psi(t)|_{L_{4}(\Omega)}+|\psi(t)|_{H_{2}^{1}(\Gamma)}\right]+\int_{0}^{t_{\max }}\left[|\mu(t)|_{H_{2}^{1}(\Omega)}^{2}+\left|\partial_{t} \psi\right|_{L_{2}(\Gamma)}^{2}\right] d t \\
& \leq C\left\{\left|\psi_{0}\right|_{H_{2}^{1}(\Omega) \cap L_{4}(\Omega) \cap H_{2}^{1}(\Gamma)}+\int_{0}^{t_{\text {max }}}\left[|f(t)|_{L_{2}(\Omega)}^{4}+|g(t)|_{L_{2}(\Gamma)}^{4}+|h(t)|_{L_{2}(\Gamma)}^{2}\right] d t\right\} .
\end{aligned}
$$

Actually, in this section we will use only $\psi \in L_{\infty}\left(J_{\max } ; H_{2}^{1}(\Omega)\right)$. The following lemma is an analogue of Lemma 3.2 and will lead to global existence.

Lemma 4.2. Suppose $p \in[2, \infty), n \leq 4$, and let $\psi \in Z(a)$.

Then there is a constant $C>0$ independent of $a>0$ such that

$$
\left\|\psi^{3}\right\|_{H_{p}^{1 / 2}\left(L_{p}\right) \cap L_{p}\left(H_{p}^{2}\right)} \leq C\|\psi\|_{Z(a)}^{\delta} \sup _{t \in J_{a}}|\psi(t)|_{H_{2}^{1}(\Omega)}^{3-\delta},
$$

where $\delta=1$ for $n=4$ and $\delta<1$ in case $n \leq 3$.

Proof. The proof is very similar to that of Lemma 3.2. We estimate as follows, using the Gagliardo-Nirenberg inequality.

$$
\left|\partial_{i} \partial_{j} \psi \psi^{2}\right|_{p} \leq\left|\partial_{i} \partial_{j} \psi\right|_{3 p}|\psi|_{3 p}^{2} \leq C\left|\nabla^{4} \psi\right|_{p}^{\alpha+2 \beta}|\psi|_{H_{2}^{1}(\Omega)}^{3-\alpha-2 \beta} \leq C|\psi|_{H_{p}^{4}(\Omega)}^{\delta} \sup _{t \in J_{a}}|\psi(t)|_{H_{2}^{1}(\Omega)}^{3-\delta},
$$

where $\delta=\alpha+2 \beta$ and $\alpha, \beta \in[0,1]$ are such that

$$
\alpha(3+n(1 / 2-1 / p)) \geq 1+n(1 / 2-1 / 3 p), \quad \beta(3+n(1 / 2-1 / p)) \geq-1+n(1 / 2-1 / 3 p) .
$$


Integrating with respect to $t$ and using Hölder's inequality, the first part follows.

Similarly,

$\left|\partial_{i} \psi \partial_{j} \psi \psi\right|_{p} \leq\left|\partial_{i} \psi\right|_{3 p}\left|\partial_{j} \psi\right|_{3 p}|\psi|_{3 p} \leq C\left|\nabla^{4} \psi\right|_{p}^{2 \alpha+\beta}|\psi|_{H_{2}^{1}(\Omega)}^{3-2 \alpha-\beta} \leq C|\psi|_{H_{p}^{4}(\Omega)}^{\delta} \sup _{t \in J_{a}}|\psi(t)|_{H_{2}^{1}(\Omega)}^{3-\delta}$,

where $\delta=2 \alpha+\beta$ and $\alpha, \beta \in[0,1]$ are such that

$$
\alpha(3+n(1 / 2-1 / p)) \geq n(1 / 2-1 / 3 p), \quad \beta(3+n(1 / 2-1 / p)) \geq-1+n(1 / 2-1 / 3 p) .
$$

Integrating with respect to $t$ and using Hölder's inequality, the second part follows.

The estimate of $\psi^{3}$ in $H_{p}^{1 / 2}\left(L_{p}\right)$ is more involved. We begin with the estimate

$$
\left\|\psi^{3}\right\|_{\left.H_{p}^{1 / 2}\left(L_{p}\right)\right)} \leq C\|\psi\|_{\left.H_{p \sigma}^{1 / 2}\left(L_{p \rho}\right)\right)}\|\psi\|_{L_{p \sigma^{\prime}}\left(L_{p \rho^{\prime}}\right)}^{2},
$$

which follows from the characterization of $H_{p}^{s}$ via differences and from Hölder's inequality; here

$$
\frac{1}{\sigma}+\frac{2}{\sigma^{\prime}}=\frac{1}{\rho}+\frac{2}{\rho^{\prime}}=1
$$

Next we use the Gagliardo-Nirenberg inequality to obtain

$$
\|\psi\|_{L_{p \sigma^{\prime}}\left(L_{p \rho^{\prime}}\right)} \leq C\|\psi\|_{Z}^{\beta}\|\psi\|_{L_{\infty}\left(H_{2}^{1 / 2}\right)}^{1-\beta},
$$

where $\beta(3+n(1 / 2-1 / p)) \geq n / 2-n / p \rho^{\prime}-1$ and $0 \leq \beta \leq 1 / \sigma^{\prime}$. We choose $\rho^{\prime}=2 n / p$ if $p \leq n$ and $\rho^{\prime}=\infty$ otherwise (then $\rho^{\prime} \in[1, \infty]$ ), and set

$$
\beta=1 / \sigma^{\prime}=\left[n-2 n / p \rho^{\prime}-2\right]_{+} / 2(3+n(1 / 2-1 / p))
$$

to meet these requirements. Next with $s=1-n / 2 p \rho^{\prime}$ we have

$$
Z \hookrightarrow H_{p}^{1-\theta}\left(J ; H_{p}^{4 \theta}(\Omega)\right) \hookrightarrow H_{p}^{s}\left(J ; L_{p \rho}(\Omega)\right)
$$

hence complex interpolation yields with $\alpha=1 / 2 s$

$$
\|\psi\|_{\left.H_{p \sigma}^{1 / 2}\left(L_{p \rho}\right)\right)} \leq C\|\psi\|_{Z}^{\alpha}\|\psi\|_{L_{p \tau}(L p \rho)}^{1-\alpha},
$$

provided

$$
1 / \sigma \geq \alpha+(1-\alpha) / \tau \text {. }
$$

Note that $s \in\{3 / 4,1\}$, hence $\alpha \in\{2 / 3,1 / 2\}$. Employing the Gagliardo-Nirenberg inequality one more time, we get

$$
\|\psi\|_{L_{p \tau}\left(L_{p \rho}\right)} \leq C|| \psi\left\|_{Z}^{\kappa}\right\| \psi \|_{L_{\infty}\left(H_{2}^{1 / 2}\right)}^{1-\kappa}
$$

with $\kappa(3+n(1 / 2-1 / p)) \geq n / 2-n / p \rho-1,0 \leq \kappa \leq 1 / \tau$.

We choose

$$
1 / \tau=\kappa=[n / 2-n / p \rho-1]_{+} /(3+n(1 / 2-1 / p)),
$$


and verify that for these choices the inequality (4.3) is valid if and only if $n \leq 4$, and the strict inequality holds if and only if $n \leq 3$, namely:

For $p \leq n$ we have $\rho^{\prime}=2 n / p, s=3 / 4, \alpha=2 / 3$, hence (4.3) is equivalent to

$$
1-\frac{[n-3]_{+}}{3+n(1 / 2-1 / p)} \geq \frac{2}{3}+\frac{n(1 / 2-1 / p)}{3(3+n(1 / 2-1 / p))}
$$

which is equivalent to

$$
[n-3]_{+} \leq 1 .
$$

For $p>n$ we have $\rho^{\prime}=\infty, s=1, \alpha=1 / 2$, hence (4.3) is equivalent to

$$
3+n(1 / 2-1 / p)-2[n-2]_{+} \geq[n(1 / 2-1 / p)-1]_{+}
$$

and the claim follows easily distinguishing the two cases of $n(1 / 2-1 / p)$ being larger than or less than 1 , respectively.

Combining these estimates, we obtain

$$
\left\|\psi^{3}\right\|_{\left.H_{p}^{1 / 2}\left(L_{p}\right)\right)} \leq C\|\psi\|_{Z}^{\delta}\|\psi\|_{L_{\infty}\left(H_{2}^{1}\right)}^{3-\delta}
$$

with

$$
\delta=2 \beta+\alpha+(1-\alpha) \kappa \leq 2 / \sigma^{\prime}+1 / \sigma=1,
$$

in fact $\delta<1$ if strict inequality holds in (4.3) which is the case if $n \leq 3$.

It is now easy to prove global existence for $p \geq 2$ and $n \leq 3$. For this purpose let $\psi$ be a solution as above. Then by maximal $L_{p}$-regularity, there is a constant $C>0$ such that

$$
\|\psi\|_{Z(a)} \leq C+C\left\|\psi^{3}\right\|_{H_{p}^{1 / 2}\left(L_{p}\right) \cap L_{p}\left(H_{p}^{2}\right)} .
$$

Using Lemmas 4.1 and 4.2 this yields

$$
\|\psi\|_{Z(a)} \leq C+C\|\psi\|_{Z(a)}^{\delta},
$$

with a different constant $C$. Now, in case $n<4$, we have $\delta<1$, which yields a bound on $\|\psi\|_{Z(a)}$ independent of $a<a_{\max }$. This in turn proves also boundedness of $\|\psi\|_{Z_{\Gamma}(a)}$, i.e. $t_{\max }=T$, hence the global existence follows. We summarize these considerations for the case $r=2-1 / p, s=0$ in

Theorem 4.3. Let $p \in[2, \infty), n \leq 3, T>0$, and let the data

$$
\begin{gathered}
\psi_{0} \in W_{p}^{4-4 / p}(\Omega) \cap W_{p}^{4-3 / p}(\Gamma), \quad f \in L_{p}(J \times \Omega) \cap L_{4}\left(J ; L_{2}(\Omega)\right), \\
g \in W_{p}^{1 / 4-1 / 4 p}\left(J ; L_{p}(\Gamma)\right) \cap L_{p}\left(J ; W_{p}^{1-1 / p}(\Gamma)\right) \cap L_{4}\left(J ; L_{2}(\Gamma)\right), \quad h \in L_{p}\left(J ; W_{p}^{2-1 / p}(\Gamma)\right),
\end{gathered}
$$

be given such that the compatibility condition

$$
\left.\partial_{\nu} \Delta \psi_{0}\right|_{\Gamma}+\left.\partial_{\nu} \psi_{0}\right|_{\Gamma}=\left.\partial_{\nu} \psi_{0}^{3}\right|_{\Gamma}+\left.g\right|_{t=0}
$$

is valid. 
Then there is a unique global solution $\psi$ of (4.1) on $J=[0, T]$ which belongs to the class

$$
\psi \in H_{p}^{1}\left(J ; L_{p}(\Omega)\right) \cap L_{p}\left(J ; H_{p}^{4}(\Omega)\right),
$$

and

$$
\left.\psi\right|_{\Gamma} \in H_{p}^{1}\left(J ; W_{p}^{2-1 / p}(\Gamma)\right) \cap L_{p}\left(J ; W_{p}^{4-1 / p}(\Gamma)\right) .
$$

In case $f, g, h$ are constant in $t$, the map $\psi_{0} \mapsto \psi(t)$ defines a global semiflow on the nonlinear phase manifold

$$
\mathcal{M}:=\left\{\psi \in W_{p}^{4-4 / p}(\Omega):\left.\psi\right|_{\Gamma} \in W_{p}^{4-3 / p}(\Gamma),\left.\partial_{\nu}\left[\Delta \psi+\psi-\psi^{3}\right]\right|_{\Gamma}=g\right\} .
$$

Note that for $p<5$ the phase manifold is the linear space $W_{p}^{4-4 / p}(\Omega) \cap W_{p}^{4-3 / p}(\Gamma)$, since for $p<5$ no compatibility conditions are involved. In particular, for $p=2$ the phase space is $H_{2}^{2}(\Omega) \cap H_{2}^{5 / 2}(\Gamma)$. Observe also that by Sobolev embedding for $p>(n+4) / 4$ $W_{p}^{4-4 / p}(\Omega)$ is a subset of the bounded continuous functions on $J \times \Omega$, in particular for $p=2$ and $n \leq 3$.

\section{$5 \quad$ Existence of Global Attractors}

In this section we shall now prove the existence of global attractor for the semiflow in

$$
M_{2}:=H^{2}(\Omega) \cap H^{2+1 / 2}(\Gamma)
$$

given by Theorem 4.3; moreover the existence of a semiflow in

$$
V:=H^{1}(\Omega) \cap H^{1}(\Gamma)
$$

together with the existence of global attractor in $V$ will be obtained simultaneously. Notice that the basic space $V$ was introduced in the work [13].

We should mention that for the Cahn-Hilliard equation subject to the usual linear boundary conditions, i.e., $\left.\partial_{\nu} \psi\right|_{\Gamma}=\left.\partial_{\nu} \Delta \psi\right|_{\Gamma}=0$, the problem of existence of global attractor has been extensively considered in the literature, e.g., in the book [14]. However, in the present paper we consider the problem of global attractor for the Cahn-Hilliard equation subject to the nonlinear and dynamical boundary condition.

First we consider the existence of a global attractor in $M_{2}$ for the problem (1.1). Based on the results in the previous section, solution to the problem (1.1) defines a semiflow in $M_{2}$. Therefore, for the existence of global attractor, we infer from Theorem 1.1 in Chapter 1 in the book [14] that it remains to verify : I. existence of an absorbing set, II. uniform compactness of the orbits.

Since we work in the $L^{2}$-setting, we use in this section the notation $\|\cdot\|$ for the $L^{2}$ norm, and $H^{k}$ for the Sobolev spaces $H_{2}^{k}$, with norm $\|\cdot\|_{H^{k}}$.

Step I, part a) Existence of an absorbing set

Let $\psi$ be the solution to the Cahn-Hilliard system

$$
\begin{aligned}
\psi_{t} & =\Delta \mu \\
\mu & =-\Delta \psi+\psi^{3}-\psi
\end{aligned}
$$




$$
\begin{gathered}
\partial_{\nu} \mu_{\mid \Gamma}=0, \quad \frac{1}{\Gamma_{s}} \psi_{t / \Gamma}=\sigma_{s} \Delta_{||} \psi-\partial_{\nu} \psi-g_{s} \psi+h \\
\psi_{\mid t=0}=\psi_{0}
\end{gathered}
$$

with $\psi_{0} \in M_{2}$. In the above $\Gamma_{s}, \sigma_{s}, g_{s}$ and $h$ are given constants.

By the boundary condition $\partial_{\nu} \mu=0$ and (5.1) we have

$$
\int_{\Omega} \psi(t, \cdot) d x=\int_{\Omega} \psi_{0} d x, \quad t \geq 0
$$

Due to this mass conservation, it is similar to the Cahn-Hilliard equation subject to the usual linear boundary conditions, we have to restrict ourselves to the metric spaces $M_{2, \gamma}$ defined below. For any given $\gamma>0$, let

$$
V_{\gamma}:=\left\{\psi \in V\left|\frac{1}{|\Omega|}\right| \int_{\Omega} \psi d x \mid \leq \gamma\right\}, \quad M_{2, \gamma}:=M_{2} \cap V_{\gamma}
$$

and let

$$
\bar{\psi}:=\frac{1}{|\Omega|} \int_{\Omega} \psi(t, \cdot) d x=\frac{1}{|\Omega|} \int_{\Omega} \psi_{0} d x .
$$

If $-\Delta_{N}$ denotes the usual realization of the negative Laplace operator in $L_{2}(\Omega)$ according to Neumann boundary conditions, a multiplication of $(5.1)$ in $L_{2}(\Omega)$ by $\left(-\Delta_{N}\right)^{-1}(\psi-\bar{\psi})$ yields

$$
\int_{\Omega} \psi_{t}\left(-\Delta_{N}\right)^{-1}(\psi-\bar{\psi}) d x+\int_{\Omega}\left(-\Delta \psi+\psi^{3}-\psi\right)(\psi-\bar{\psi}) d x=0
$$

Introducing $H_{*}^{1}:=\left\{v \in H^{1}(\Omega) \mid \int_{\Omega} v d x=0\right\}$ with inner product $\langle u, v\rangle_{*}:=\int_{\Omega} \nabla u \nabla v d x$, the norm $\|\cdot\|_{-1}$ given by

$$
\|h\|_{-1}:=\left(\int_{\Omega}\left(-\Delta_{N}\right)^{-1} h h d x\right)^{1 / 2}
$$

is just the norm in the dual space $H^{-1}:=\left(H_{*}^{1}\right)^{*}$. We then obtain, using (5.3),

$$
\begin{aligned}
& \frac{1}{2} \frac{d}{d t}\|\psi-\bar{\psi}\|_{-1}^{2}+\int_{\Omega}\left(|\nabla \psi|^{2}+\psi^{4}-\psi^{2}\right) d x+\int_{\Gamma}\left(\sigma_{s}\left|\nabla_{||} \psi\right|^{2}+g_{s} \psi^{2}-h \psi\right) d s \\
= & -\frac{1}{\Gamma_{s}} \int_{\Gamma} \psi_{t}(\psi-\bar{\psi}) d s+\int_{\Omega}\left(\psi^{3}-\psi\right) \bar{\psi} d x+\int_{\Gamma}\left(g_{s} \psi-h\right) \bar{\psi} d s
\end{aligned}
$$

Recalling from [13] the relevant energy functional (cp. (4.2))

$$
F[\psi]:=\int_{\Omega}\left[\frac{1}{2}|\nabla \psi|^{2}+\frac{1}{4} \psi^{4}-\frac{1}{2} \psi^{2}\right] d x+\int_{\Gamma}\left[\frac{\sigma_{s}}{2}\left|\nabla_{\|} \psi\right|^{2}+\frac{g_{s}}{2} \psi^{2}-h \psi\right] d s,
$$

we have

$$
\frac{d}{d t} F[\psi]+\|\nabla \mu\|^{2}+\frac{1}{\Gamma_{s}} \int_{\Gamma}\left|\psi_{t}\right|^{2}=0
$$


where, as mentioned before, $\|\cdot\|$ will denote the $L_{2}(\Omega)$-norm in this section. For small $\eta>0$ to be specified later, we multiply (5.5) by $\eta$ and add it to (5.7), to the result

$$
\begin{aligned}
& \frac{d}{d t}\left\{F[\psi]+\frac{\eta}{2}\|\psi-\bar{\psi}\|_{-1}^{2}\right\}+\|\nabla \mu\|^{2}+\frac{1}{\Gamma_{s}} \int_{\Gamma} \psi_{t}^{2} d s \\
& +\eta \int_{\Omega}\left[|\nabla \psi|^{2}+\psi^{4}-\psi^{2}\right] d x+\eta \int_{\Gamma}\left[\sigma_{s}\left|\nabla_{\|} \psi\right|^{2}+g_{s} \psi^{2}-h \psi\right] d x \\
& \quad=-\frac{\eta}{\Gamma_{s}} \int_{\Gamma}(\psi-\bar{\psi}) \psi_{t} d s+\eta \int_{\Omega}\left(\psi^{3}-\psi\right) \bar{\psi} d x+\eta \int_{\Gamma}\left(g_{s} \psi-h\right) \bar{\psi} d s .
\end{aligned}
$$

Using Young's inequality we have

$$
\begin{aligned}
-\frac{\eta}{\Gamma_{s}} \int_{\Gamma}(\psi-\bar{\psi}) \psi_{t} d s & \leq \frac{1}{2 \Gamma_{s}} \int_{\Gamma} \psi_{t}^{2} d s+\int_{\Gamma} \frac{\eta^{2}}{2 \Gamma_{s}}|\psi-\bar{\psi}|^{2} d s \\
\int_{\Omega}\left(\psi^{3}-\psi\right) \bar{\psi} d x & \leq \frac{1}{4} \int_{\Omega} \psi^{4} d x+C_{\gamma}
\end{aligned}
$$

where $C_{\gamma}$ denotes here and in the sequel a positive constant depending only on $\gamma$ and $\Omega$. Similarly, we have

$$
\int_{\Gamma}\left(g_{s} \psi-h\right) \bar{\psi} d s \leq \int_{\Gamma} \frac{g_{s}}{4} \psi^{2} d s+C_{\gamma}
$$

Let

$$
\eta:=\frac{\Gamma_{s} g_{s}}{4}
$$

Then we deduce from (5.8) that

$$
\frac{d}{d t}\left\{F[\psi]+\frac{\eta}{2}\|\psi-\bar{\psi}\|_{-1}^{2}\right\}+\eta F[\psi]+\frac{\eta}{4} \int_{\Omega} \psi^{4} d x \leq C_{\gamma}
$$

Notice that

$$
\|\psi-\bar{\psi}\|_{-1}^{2} \leq C\|\psi-\bar{\psi}\|^{2} \leq \frac{1}{2 \eta} \int_{\Gamma} \psi^{4} d x+C_{\gamma}
$$

where $C$ denotes here and in the sequel a positive constant depending only on $\Omega$. Thus,

$$
\frac{d}{d t}\left\{F[\psi]+\frac{\eta}{2}\|\psi-\bar{\psi}\|_{-1}^{2}\right\}+\eta\left\{F[\psi]+\frac{\eta}{2}\|\psi-\bar{\psi}\|_{-1}^{2}\right\} \leq C_{\gamma}
$$

which implies

$$
F[\psi]+\frac{\eta}{2}\|\psi-\bar{\psi}\|_{-1}^{2} \leq e^{-\eta t}\left(F\left[\psi_{0}\right]+\frac{\eta}{2}\left\|\psi_{0}-\bar{\psi}\right\|_{-1}^{2}\right)+\frac{C_{\gamma}}{\eta} .
$$

Since

$$
F[\psi] \geq \frac{1}{2}\|\psi\|_{V}^{2}-C,
$$

we conclude that there is a ball $B_{0}$ in $V_{\gamma}$ with its radius depending only on $\gamma, \Omega$ such that for any initial data in $M_{2, \gamma}$ which are in a $V$-bounded subset $B \subset V_{\gamma}$ there is $t_{1}=t_{1}(B)>0$ such that for all $t \geq t_{1}$ the orbits enter into $B_{0}$.

Before continuing in showing the existence of an absorbing set in $M_{2, \gamma}$, we turn to the question of uniform compactness. 
Step II: Uniform compactness of the orbits.

It follows from (5.7) that

$$
F[\psi]+\int_{0}^{t}\|\nabla \mu\|^{2} d \tau+\frac{1}{\Gamma_{s}} \int_{0}^{t} \int_{\Gamma} \psi_{t}^{2} d s d \tau=F\left[\psi_{0}\right]
$$

For initial data varying in a given bounded set of $V_{\gamma}$ we have

$$
F\left[\psi_{0}\right] \leq C
$$

where $C>0$ depends only on $\left\|\psi_{0}\right\|_{V}$.

Thus

$$
\begin{aligned}
\int_{0}^{t}\|\nabla \mu\|^{2} d \tau & \leq C, \quad \int_{0}^{t} \int_{\Gamma} \psi_{t}^{2} d s d \tau \leq C \\
F[\psi(t)] & \leq C, \quad\|\psi(t)\|_{V} \leq C
\end{aligned}
$$

Differentiating (5.2) with respect to $t$, we obtain

$$
\mu_{t}=-\Delta \psi_{t}+3 \psi^{2} \psi_{t}-\psi_{t} .
$$

Multiplying (5.21) by $\psi_{t}$ and integrating over $\Omega$ yields

$$
\begin{gathered}
\frac{1}{2} \frac{d}{d t}\|\nabla \mu\|^{2}+\left\|\nabla \psi_{t}\right\|^{2}+\int_{\Omega} 3 \psi^{2} \psi_{t}^{2} d x+\frac{1}{2 \Gamma_{s}} \frac{d}{d t} \int_{\Gamma} \psi_{t}^{2} d s+\int_{\Gamma}\left[\sigma_{s}\left|\nabla_{\|} \psi_{t}\right|^{2}+g_{s} \psi_{t}^{2}\right] d s \\
\quad=\int_{\Omega} \psi_{t}^{2} d x=\int_{\Omega} \Delta \mu \psi_{t} d x=-\int_{\Omega} \nabla \mu \nabla \psi_{t} d x \\
\leq \frac{1}{2}\left\|\nabla \psi_{t}\right\|^{2}+\frac{1}{2}\|\nabla \mu\|^{2}
\end{gathered}
$$

Multiplying (5.22) by $t$ and integrating with respect to $t$, we obtain that

$$
\begin{aligned}
t\left(\|\nabla \mu\|^{2}\right. & \left.+\frac{1}{\Gamma_{s}} \int_{\Gamma} \psi_{t}^{2} d s\right)+\int_{0}^{t} \tau\left(\left\|\nabla \psi_{t}\right\|^{2}+\int_{\Omega} \psi^{2} \psi_{t}^{2} d x+\int_{\Gamma}\left[\sigma_{s}\left|\nabla_{\|} \psi_{t}\right|^{2}+g_{s} \psi_{t}^{2}\right] d s\right) d \tau \\
& \leq C\left(\int_{0}^{t} \tau\|\nabla \mu\|^{2} d \tau+\int_{0}^{t}\left[\|\nabla \mu\|^{2}+\int_{\Gamma} \psi_{t}^{2} d s\right] d \tau\right) \\
& \leq C\left(t \int_{0}^{t}\|\nabla \mu\|^{2}+\int_{0}^{t}\left[\|\nabla \mu\|^{2}+\int_{\Gamma} \psi_{t}^{2} d s\right] d \tau\right)
\end{aligned}
$$

Thus, by (5.19), for $t>0$,

$$
\|\nabla \mu(t)\|^{2}+\frac{1}{\Gamma_{s}} \int_{\Gamma} \psi_{t}^{2} d s \leq C\left(1+\frac{1}{t}\right)
$$

where $C$ in this Step II always denotes a positive constant depending at most on $\left\|\psi_{0}\right\|_{V}$. On the other hand, integration of (5.18) over $\Omega$ yields that

$$
\int_{\Omega} \mu d x=\int_{\Omega}\left[\psi^{3}-\psi\right] d x+\int_{\Gamma}\left[\frac{1}{\Gamma_{s}} \psi_{t}+g_{s} \psi-h\right] d s .
$$


Using Young's inequality, we conclude from (5.20), (5.24), (5.25) that for $t \geq \delta>0$,

$$
\left|\int_{\Omega} \mu d x\right| \leq C_{\delta}
$$

where $C_{\delta}$ denotes here and in the sequel a positive constant depending only on $\left\|\psi_{0}\right\|_{V}$ and on $\delta$.

It also follows from (5.23) that for $t>0$,

$$
\frac{1}{t} \int_{0}^{t} \tau\left(\left\|\nabla \psi_{t}\right\|^{2}+\int_{\Omega} \psi^{2} \psi_{t}^{2} d x+\int_{\Gamma}\left[\sigma_{s}\left|\nabla_{\Gamma} \psi_{t}\right|^{2}+g_{s} \psi_{t}^{2}\right] d s\right) d \tau \leq C\left(1+\frac{1}{t}\right) .
$$

Differentiating (5.2) with respect to $t$ and multiplying by $\psi_{t t}$ in $L_{2}(\Omega)$ yields

$$
\begin{gathered}
\frac{1}{2} \frac{d}{d t}\left\|\nabla \psi_{t}\right\|^{2}-\frac{1}{2} \frac{d}{d t}\left\|\psi_{t}\right\|^{2}+\frac{3}{2} \frac{d}{d t} \int_{\Omega} \psi^{2} \psi_{t}^{2} d x+\frac{1}{2} \frac{d}{d t} \int_{\Gamma}\left[\sigma_{s}|\nabla|\left|\psi_{t}\right|^{2}+g_{s} \psi_{t}^{2}\right] d s \\
+\left\|\nabla \mu_{t}\right\|^{2}+\frac{1}{\Gamma_{s}} \int_{\Gamma} \psi_{t t}^{2} d s=3 \int_{\Omega} \psi \psi_{t}^{3} d x
\end{gathered}
$$

Since $n \leq 3$, we obtain by (5.20) that

$$
\left|\int_{\Omega} \psi \psi_{t}^{3} d x\right| \leq C\left\|\psi_{t}\right\|_{L_{\frac{18}{5}}}
$$

Using the Gagliardo-Nirenberg inequality, we get

$$
\left\|\psi_{t}\right\|_{L_{\frac{18}{5}}} \leq C\left(\left\|\psi_{t}\right\|_{H^{1}}^{2}\left\|\psi_{t}\right\|+\left\|\psi_{t}\right\|^{3}\right)
$$

Multiplying (5.28) by $t^{2}$ and integrating with respect to $t$, we obtain

$$
\begin{aligned}
t^{2}\left\{\frac{1}{2}\left\|\nabla \psi_{t}\right\|^{2}-\frac{1}{2}\left\|\psi_{t}\right\|^{2}+\frac{3}{2} \int_{\Omega} \psi^{2} \psi_{t}^{2} d x+\frac{1}{2} \int_{\Gamma}\left[\sigma_{s}\left|\nabla_{\|} \psi_{t}\right|^{2}+g_{s} \psi_{t}^{2}\right] d s\right. \\
\left.\left.+\int_{0}^{t} \tau^{2}\left\|\nabla \mu_{t}\right\|^{2}+\frac{1}{\Gamma_{s}} \int_{\Gamma} \psi_{t t}^{2} d s\right] d \tau\right\}+\int_{0}^{t} \tau\left\|\psi_{t}\right\|^{2} d \tau \\
=\int_{0}^{t} \tau\left(\left\|\nabla \psi_{t}\right\|^{2}+3 \int_{\Omega} \psi^{2} \psi_{t}^{2} d x+\int_{\Gamma}\left[\sigma_{s}\left|\nabla_{\|} \psi_{t}\right|^{2}+g_{s} \psi_{t}^{2}\right] d s\right) d \tau \\
+3 \int_{0}^{t} \tau^{2} \int_{\Omega} \psi \psi_{t}^{3} d x d \tau
\end{aligned}
$$

i.e., by $(5.29)$,

$$
\begin{aligned}
t^{2}\left\{\frac{1}{2} \|\right. & \nabla \psi_{t} \|^{2}+\frac{3}{2} \int_{\Omega} \psi^{2} \psi_{t}^{2} d x+\frac{1}{2} \int_{\Gamma}\left[\sigma_{s}\left|\nabla_{\|} \psi_{t}\right|^{2}+g_{s} \psi_{t}^{2}\right] d s \\
& \left.+\int_{0}^{t} \tau^{2}\left[\left\|\nabla \mu_{t}\right\|^{2}+\frac{1}{\Gamma_{s}} \int_{\Gamma} \psi_{t t}^{2} d s\right] d \tau\right\}+\int_{0}^{t} \tau\left\|\psi_{t}\right\|^{2} d \tau \\
& \leq \frac{t^{2}}{2}\left\|\psi_{t}\right\|^{2}+\int_{0}^{t} \tau\left(\left\|\nabla \psi_{t}\right\|^{2}+3 \int_{\Omega} \psi^{2} \psi_{t}^{2} d x+\int_{\Gamma}\left[\sigma_{s}\left|\nabla_{\|} \psi_{t}\right|^{2}+g_{s} \psi_{t}^{2}\right] d s\right) d \tau \\
& +C \int_{0}^{t} \tau^{2}\left[\left\|\psi_{t}\right\|_{H^{1}}^{2}\left\|\psi_{t}\right\|+\left\|\psi_{t}\right\|^{3}\right] d \tau
\end{aligned}
$$


Using again

$$
\left\|\psi_{t}\right\|^{2}=\int_{\Omega} \psi_{t} \Delta \mu d x=-\int_{\Omega} \nabla \psi_{t} \nabla \mu d x \leq\left\|\nabla \psi_{t}\right\|\|\nabla \mu\|,
$$

we have by (5.24)

$$
\frac{t^{2}}{2}\left\|\psi_{t}\right\|^{2} \leq \frac{t^{2}}{4}\left\|\nabla \psi_{t}\right\|^{2}+C t^{2}\left(1+\frac{1}{t}\right)
$$

Moreover,

$$
\begin{aligned}
& \int_{0}^{t} \tau^{2}\left\|\psi_{t}\right\|_{H^{1}}^{2}\left\|\psi_{t}\right\| d \tau \leq \sup _{0 \leq \tau \leq t}\left(\tau\left\|\psi_{t}(\tau)\right\|_{H^{1}}\right)\left(\int_{0}^{t} \tau\left\|\psi_{t}\right\|_{H^{1}}^{2} d \tau\right)^{\frac{1}{2}}\left(\int_{0}^{t} \tau\left\|\psi_{t}\right\|^{2} d \tau\right)^{\frac{1}{2}} \\
& \int_{0}^{t} \tau\left\|\psi_{t}\right\|^{2} d \tau \leq \int_{0}^{t} \tau\left\|\nabla \psi_{t}\right\|\|\nabla \mu\| d \tau \leq t^{\frac{1}{2}}\left(\int_{0}^{t} \tau\left\|\nabla \psi_{t}\right\|^{2} d \tau\right)^{\frac{1}{2}}\left(\int_{0}^{t}\|\nabla \mu\|^{2} d \tau\right)^{\frac{1}{2}} \\
& \leq C t^{\frac{1}{2}}\left(\int_{0}^{t} \tau\left\|\nabla \psi_{t}\right\|^{2} d \tau\right)^{\frac{1}{2}}
\end{aligned}
$$

where we have used (5.19).

It follows from (5.27), (5.34), (5.35) that

$$
\begin{aligned}
\int_{0}^{t} \tau^{2}\left\|\psi_{t}\right\|_{H^{1}}^{2}\left\|\psi_{t}\right\| d \tau & \leq \sup _{0 \leq \tau \leq t}\left(\tau\left\|\psi_{t}(\tau)\right\|_{H^{1}}\right) C t\left(1+\frac{1}{t}\right) \\
& \leq \varepsilon \sup _{0 \leq \tau \leq t} \tau^{2}\left\|\psi_{t}(\tau)\right\|_{H^{1}}^{2}+\frac{C}{\varepsilon} t^{2}\left(1+\frac{1}{t}\right)^{2}
\end{aligned}
$$

with $\varepsilon>0$ being a small constant to be specified below.

Thus we obtain from (5.31)-(5.36), using (5.27), for $t>0$

$$
\begin{gathered}
t^{2}\left\{\frac{1}{4}\left\|\nabla \psi_{t}\right\|^{2}+\frac{3}{2} \int_{\Omega} \psi^{2} \psi_{t}^{2} d x+\frac{1}{2} \int_{\Gamma}\left[\sigma_{s}\left|\nabla_{\|} \psi_{t}\right|^{2}+g_{s} \psi_{t}^{2}\right] d s\right\} \\
\leq C t^{2}\left(1+\frac{1}{t}\right)+C t\left(1+\frac{1}{t}\right)+\frac{C}{\varepsilon} t^{2}\left(1+\frac{1}{t}\right)^{2} \\
+\varepsilon \sup _{0 \leq \tau \leq t}\left(\tau^{2}\left\|\psi_{t}(\tau)\right\|_{H^{1}}^{2}\right) .
\end{gathered}
$$

Taking the supremum with respect to $t$ from 0 to $t$ and taking $\varepsilon$ small enough and fixed now, we deduce from (5.37) that

$$
\sup _{0 \leq \tau \leq t}\left(\tau^{2}\left\|\psi_{t}(\tau)\right\|_{H^{1}}^{2} \leq C t^{2}\left(1+\frac{1}{t}\right)+C t\left(1+\frac{1}{t}\right)+C t^{2}\left(1+\frac{1}{t}\right)^{2} .\right.
$$

Thus it follows from (5.37) and (5.38) that for $t \geq \delta>0$, where $\delta<1$ without loss of generality,

$$
\left\|\nabla \psi_{t}(t)\right\|^{2}+\int_{\Omega} \psi^{2} \psi_{t}^{2} d x+\int_{\Gamma}\left[\sigma_{s}\left(\left.\nabla_{\|} \psi_{t}\right|^{2}+g_{s} \psi_{t}^{2}\right] d s \leq \frac{C}{\delta}\left(1+\frac{1}{\delta}\right) .\right.
$$


By the elliptic estimate (cp. [13, (4.11)])

$$
\|\psi(t)\|_{H^{3}} \leq C\left(\|\mu\|_{H^{1}}+\left\|\psi_{t}\right\|_{H^{1 / 2}(\Gamma)}+\|h\|_{H^{1 / 2}(\Gamma)}\right),
$$

we obtain, using (5.24), (5.26), (5.39), for $t \geq \delta>0$,

$$
\|\psi(t)\|_{H^{3}} \leq C_{\delta}
$$

with $C_{\delta}$ being a positive constant depending only on $\delta, \gamma$ and the norm of initial data in $V$. This means that for initial data belonging to $M_{2}$ and staying in a bounded subset of $V_{\gamma}$, for $t \geq \delta>0$ the orbits are uniformly bounded in $H_{3}(\Omega)$. To prove the uniform compactness in $M_{2, \gamma}=H^{2}(\Omega) \cap H^{2+1 / 2}(\Gamma) \cap V_{\gamma}$ for $t \geq \delta>0$, it remains to prove that $\|\psi(t)\|_{H^{3}(\Gamma)}$ is also uniformly bounded. From (5.39) we can conclude that for $t \geq \delta>0$,

$$
\left\|\psi_{t}(t)\right\|_{H^{1}(\Gamma)} \leq C_{\delta} .
$$

Thus, from the boundary condition (5.3) we have

$$
\sigma_{s} \Delta_{\|} \psi=\frac{1}{\Gamma_{s}} \psi_{t}+\partial_{\nu} \psi+\sigma_{s} \psi-h \in H_{1}(\Gamma)
$$

By elliptic regularity for $\Delta_{\|}$, we get for $t \geq \delta>0$,

$$
\begin{aligned}
\|\psi(t)\|_{H^{3}(\Gamma)} & \leq C\left(\left\|\psi_{t}(t)\right\|_{H^{1}(\Gamma)}+\left\|\partial_{\nu} \psi(t)\right\|_{H^{1}(\Gamma)}+\|\psi(t)\|_{H^{1}(\Gamma)}+C\right) \\
& \leq C_{\delta}
\end{aligned}
$$

due to $(5.40),(5.41)$.

Thus (5.40) and (5.42) imply the uniform compactness of the orbits in $M_{2, \gamma}$ for $t \geq \delta>0$.

Step I - part b): Existence of an absorbing set in $M_{2, \gamma}$

For initial data in any given bounded set in $M_{2}$, which naturally are in a bounded set $B$ in $V$, by (5.15), (5.16), there is a ball $B_{0}$ in $V$ with its radius $r_{B_{0}}$ depending only on $\gamma$, and $t_{1}(B)$ such that for $t \geq t_{1}$, the orbits enter $B_{0}$. Now by (5.40), for $t \geq t_{1}+\delta>0$ with $\delta$ being fixed, e.g., $\delta=1$,

$$
\|\psi(t)\|_{M_{2}} \leq C_{\delta},
$$

$C_{\delta}$ depending only on $r_{B_{0}}$ and $\delta$. Thus, the existence of an absorbing set in $M_{2, \gamma}$ follows. Combining the existence of an absorbing set in $M_{2, \gamma}$ and the uniform compactness of orbits in $M_{2, \gamma}$ proved in Step I and Step II, respectively, by Theorem 1.1 in Chapter 1 in the book [14], we have proved

Theorem 5.1. For any $\gamma>0$ there is a global attractor $\mathcal{A}_{\gamma}$ in $M_{2, \gamma}$ for the semiflow associated to the Cahn-Hilliard system (5.1)-(5.4).

Finally we want to prove that for initial data in $V$ the Cahn-Hilliard system (5.1)-(5.4) admits a unique global solution which also defines a semiflow in $V$. This was not known 
in the previous work by Racke and Zheng [13]. This is also an essential step towards a proof of the existence of a global attractor.

Since $M_{2}$ is dense in $V$, for any initial data in $V$ there is a sequence of initial data in $M_{2}$ converging to the given initial data in $V$. Therefore, by the results in the previous section, there is a sequence of approximate solutions which belong to $M_{2}$ for $t \geq 0$. By a similar discussion as in the proof of the uniform compactness in $M_{2}$ above, we can conclude that the approximate solutions will converge in $M_{2}$ for any $t>0$. Thus the limit is a global solution to the Cahn-Hilliard system (5.1)-(5.4). The uniqueness can also be easily proved by the energy method. In a similar way as deriving (5.7), we can easily show that the solution is also continuous in $V$ for $t \geq 0$. Thus the solutions defines a continuous semiflow in $V$. From (5.15), (5.16), (5.40) we can conclude the existence of an absorbing ball in $V_{\gamma}$ as well as the uniform compactness of the orbits in $V_{\gamma}$ for $t \geq \delta>0$. Thus we have proved the following result.

Theorem 5.2. The solution for the Cahn-Hilliard system (5.1)-(5.4) defines a continuous semiflow in $V$. Moreover, for any $\gamma>0$ there is a global attractor $\mathcal{A}_{\gamma}$ in $V_{\gamma}$.

\section{References}

[1] H. Amann, Linear and Quasilinear Parabolic Problems, Volume I, Abstract Linear Theory Monographs in Mathematics, Birkhäuser, Basel, 1995.

[2] J.W. Cahn and E. Hilliard, Free energy of a nonuniform system, I, Interfacial free energy, J. Chem. Phys. 28(1958), 258-367.

[3] R. Denk, M. Hieber, and J. Prüss. $\mathcal{R}$-boundedness and problems of elliptic and parabolic type. Memoirs Amer. Math. Soc., to appear, 2003.

[4] R. Denk, M. Hieber, and J. Prüss. Optimal $L_{p}-L_{q}$-regularity for vector-valued parabolic problems with inhomogeneous boundary data. submitted, 2003.

[5] M. Hieber and J. Prüss. Heat kernels and maximal $L_{p}-L_{q}$ estimates for parabolic evolution equations. Comm. Partial Differential Equations, 22:1647-1669, 1997.

[6] J. Escher, J. Prüss, and G. Simonett. A new approach to the regularity of solutions for parabolic equations. em Evolution Equations, eds. G. Goldstein, R. Nagel, S. Romanelli, Marcel Dekker, New York, 2003.

[7] M. Hieber and J. Prüss. Maximal Regularity of Parabolic Problems. Monograph in Preparation, 2003.

[8] R. Kenzler, F. Eurich, P. Maass, B. Rinn, J. Schropp, E. Bohl, and W. Dieterich, Phase separation in confined geometries: Solving the Cahn-Hilliard equation with generic boundary conditions, Computer Phys. Comm. 133(2001), 139-157.

[9] O.A. Ladyžhenskaya, V.N. Solonnikov, N.N. Uralt'seva, Linear and Quasilinear Equations of Parabolic Type. Izdat. Nauka, Moskow, 1968.

[10] A. Novick-Cohen and L.A. Segel, Nonlinear aspects of the Cahn-Hilliard equation, Physica D, 10(1984), 277-298.

[11] J. Prüss, Evolutionary Integral Equations and Applications. Monographs in Mathematics, Birkhäuser, Basel, 1993.

[12] J. Prüss. Maximal regularity for abstract parabolic problems with inhomogeneous boundary data. Math. Bohemica, 127:311-327, 2002.

[13] R. Racke and S. Zheng. The Cahn-Hilliard equation with dynamic boundary conditions. Advances Diff. Equations, 8:83-110, 2003.

[14] R. Temam. Infinite-Dimensional Dynamical Systems in Mechanics and Physics. Appl. Math. Sci. 68, Springer-Verlag, New York, 1988. 